\section{Neue Schätzung zur Krankheitslast durch Krankenhaus-Infektionen}

Das Robert Koch-Institut hat zusammen mit Partnern aus Berlin und Stockholm Ende 2019 eine aktuelle Schätzung zur Krankheitslast durch im Krankenhaus erworbene (nosokomiale) Infektionen vorgelegt.

„Damit können wir die Auswirkungen nosokomialer Infektionen auf die Gesundheit der Bevölkerung genauer abbilden und verlässlichere evidenzbasierte Grundlagen für Maßnahmen bereitstellen“, betont Lothar H. Wieler, Präsident des RKI. Die Studie entstand in Zusammenarbeit mit dem Europäischen Zentrum für die Prävention und die Kontrolle von Krankheiten und dem Nationalen Referenzzentrum für die Surveillance nosokomialer Infektionen an der Charité Universitätsmedizin Berlin. Die Ergebnisse sind in der Fachzeitschrift Eurosurveillance (46/2019) erschienen.

Die in der neuen Studie geschätzte Zahl der nosokomialen Infektionen in Deutschland liegt bei 400000 bis 600000 pro Jahr und damit im Bereich vorheriger Hochrechnungen. Die Zahl der Todesfälle kann durch die weiterentwickelte Methodik verlässlicher erfasst werden und liegt jetzt bei 10000 bis 20 000.

Der Anteil der Patienten, die während eines Krankenhaus-Aufenthaltes eine Infektion bekommen, ist in Deutschland mit rund 3,6\% niedriger als im EU-Durchschnitt $(5,5 \%)$. Betrachtet man aus Public-Health-Sicht die Krankheitslast auf Bevölkerungsebene, ergibt sich ein anderes Bild. Bezogen auf die Bevölkerung liegt Deutschland bei der Zahl der Infektionen und Todesfälle sowie der Krankheitslast (DALY) über dem europäischen Schnitt. So erkranken hierzulande jährlich 500 bis 650 Patienten pro 100000 Einwohner an einer nosokomialen Infektion, im EU Durchschnitt sind es 450 bis 500 Erkrankte pro 100000 Einwohner. Derartige Zahlen können nicht isoliert betrachtet werden, bei einem Vergleich müssen die unterschiedlichen Gesundheitssysteme in den europäischen Ländern mitberücksichtigt werden.

Eine wesentliche Ursache für die höhere Krankheitslast in Deutschland ist die gröBere Zahl an stationär behandelten Patienten und Krankenhausbetten. Deutschland hat in Europa die höchste Anzahl an Krankenhausbetten und die zweithöchste Anzahl an Krankenhauspatienten pro 1000 Einwohner und Jahr. „Eine Reduktion vermeidbarer Krankenhausaufenthalte sind daher zusammen mit einer effektiven Infektionskontrolle und -prävention wichtige Schritte, um die Krankheitslast zu verringern", unterstreicht Lothar Wieler.

Die Wissenschaftler haben für die neue Studie 5 Infektionen betrachtet, die fast $80 \%$ der im Krankenhaus erworbenen Infektionen ausmachen, Lungenentzündungen, Harnwegsinfektionen, Wundinfektionen, Clostridium difficile-Infektionen und Blutstrominfektionen. Die Daten stammen aus der sogenannten Punktprävalenzstudie 2011/2012 des Nationalen Referenzzentrums.

[1] Benedikt Zacher et al: Application of a new methodology and $R$ package reveals a high burden of healthcare-associated infections (HAl) in Germany compared to the average in the European Union/European Economic Area, 2011 to 2012 www.eurosurveillance.org (Ausgabe 46 vom 14.11.2019)

Nach einer Mitteilung des Robert KochInstituts 